\title{
Pneumocystis carinii pneumonia: detection of parasites in sputum and bronchoalveolar lavage fluid by monoclonal antibodies
}

\author{
Kerstin M Elvin, Anders Björkman, Ewert Linder, Nadja Heurlin, Anders Hjerpe
}

\begin{abstract}
Diagnosis of pneumocystis pneumonia is based on identifying Pneumocystis carinii cytochemically in material from the lung. The silver methenamine staining methods most commonly used are technically difficult and lack specificity. The diagnostic value of immunocytological identification of the parasite was evaluated by using mouse monoclonal antibody 3 F6, specific for human pneumocystis, to identify $P$ carinii in bronchoalveolar lavage fluid and sputum by immunofluorescence and was compared with that of other variables.

Bronchoalveolar lavage was performed on 25 patients positive for HIV antibody with clinically suspected pneumocystis pneumonia and 40 patients negative for HIV antibody who presented with interstitial disorders of the lung. Lavage fluid showed pneumocystis only in the patients positive for antibody, the parasite being detected in 19 by immunofluorescence and in $\mathbf{1 7}$ by a modified silver methenamine staining method. Chest $x$ ray films obtained at the time of bronchoscopy showed interstitial or alveolar shadowing in 17 of the 19 patients, but clinical symptoms and the presence of antibodies to pneumocystis did not seem to be predictive.

Sputum samples were collected during 43 episodes of clinically suspected pneumocystis pneumonia in patients positive for HIV antibody. Pneumocystis was detected consistently more commonly by immunofluorescence than the silver strain in sputum collected routinely and induced by inhalation of saline. In 17 patients bronchoalveolar lavage followed sputum collection, and the sensitivity of detection of pneumocystis in immunofluorescence in sputum compared with lavage fluid was $57 \%$ (8/14).

Immunofluorescence was suitable for specimens fixed in ethanol and seemed highly specific and more sensitive than the standard cytochemical methods for identifying pneumocystis.
\end{abstract}

Parasitology, National

Bacteriological

Laboratory, S-105 21

Stockholm, Sweden

Kerstin M Elvin, MD, research clinician

Anders Björkman, MD, research clinician

Ewert Linder, MD, assistant professor

Huddinge Hospital, Huddinge, S-14186, Sweden

Nadja Heurlin, MD, senior registrar, department of lung medicine

Anders Hjerpe, $\mathrm{MD}$, assistant professor, department of pathology

\section{Introduction}

Pneumocystis carinii has been increasingly recognised as an important cause of opportunistic infection in immunodeficient hosts. Pneumocystis pneumonia is the most common treatable infection in patients with AIDS, occurring in over $60 \% .^{.}$The diagnosis is based on visual detection of the parasite in material from the lung. Open lung biopsy was previously the preferred procedure for recovering the organism but has been replaced by bronchoscopy combined with bronchoalveolar lavage or transbronchial biopsy, or both, which have proved to be as sensitive and less traumatic. ${ }^{23}$ Recent evaluation of cytochemical staining of specimens of induced sputum showed a sensitivity of about $55 \%$. $^{+5}$

Several cytological stains have been used for identi- fying pneumocystis. ${ }^{6}$ Different modifications of Gomori's silver methenamine staining method have been commonly used, ${ }^{7}$ but these may be technically difficult, the microscopic examination may be time consuming, and the presence of, for example, silver stained fungi and argyrophilic tissue elements may cause diagnostic problems. ${ }^{78}$ These problems together with the increased demand for diagnosis of pneumocystis pneumonia and for safe handling of samples from patients with human immunodeficiency virus (HIV) require that the method of detection be improved. Immunocytological staining of pneumocystis in bronchoalveolar lavage fluid and lung tissue from experimentally infected rats was shown to be better than cytochemical methods, ${ }^{910}$ the advantages including earlier detection of the parasite, more rapid enumeration, and detection of small trophozoites and free forms. Monoclonal antibodies for specifically identifying pneumocystis in material from the lung have therefore been developed."

We have developed a mouse monoclonal antibody to human pneumocystis (3F6) that can detect the parasite in fixed samples in which HIV has been inactivated. ${ }^{12} 13$ Immunocytological staining with this antibody did not show any reactivity with cryptosporidium, Aspergillus fumigatus, six Candida species, or several protozoa.

The aim of this study was to evaluate the ability of this monoclonal antibody to detect the parasite in bronchoalveolar lavage fluid and sputum that had been fixed with ethanol. The diagnostic value of clinical symptoms, chest $x$ ray films, and the presence of antibodies to pneumocystis was also evaluated.

\section{Patients and methods}

We obtained samples from patients admitted to Roslagstull, Danderyd, and Huddinge Hospitals in Stockholm. Because of practical and clinical circumstances both sputum and bronchoalveolar lavage fluid could not always be obtained within a reasonable time from all patients.

We studied three groups of patients, two of which 
stitial disorders of the lung in whom immunodeficiency was not known or clinically suspected.

We obtained all sputum samples during 1986 from patients positive for HIV antibody with suspected pneumocystis pneumonia admitted to Roslagstull and Danderyd hospitals. A total of 43 suspected episodes of pneumocystis pneumonia occurred in 34 patients (17 of whom were included in the study group), nine patients having two separate episodes more than three months apart.

Bronchoalveolar lavage - The bronchial tree was examined to subsegmental level by fibreoptic bronchoscopy and the bronchoscope wedged into the most suitable subsegment. A total of $150-200 \mathrm{ml}$ lukewarm saline at $37^{\circ} \mathrm{C}$ was instilled in $50 \mathrm{ml}$ aliquots, and each aliquot was aspirated. Usually $50-100 \mathrm{ml}$ of fluid was recovered." Additional material for cytological examination was obtained simultaneously by bronchial brushing, and transbronchial biopsy specimens were taken in many cases.

Sputum collection-Routine sputum samples were collected (if necessary with the help of a physiotherapist) and treated with an equal amount of $6.5 \mathrm{mM}$ dithiothreitol (Behring Diagnostics). An equal volume of $50 \%(\mathrm{v} / \mathrm{v})$ ethanol was added before the specimen was transported to the laboratory. ${ }^{15}{ }^{16}$ Induced sputum was obtained after patients had inhaled a mist of saline generated by a nebuliser for about 10 minutes. ${ }^{+}$The samples were treated as described above.

Histochemical staining for detecting pneumocystisBronchoalveolar lavage fluid was fixed with $50 \%(\mathrm{v} / \mathrm{v})$ ethanol and centrifuged, and smears were prepared from the pellets. Biopsy specimens were fixed with $4 \%(\mathrm{v} / \mathrm{v})$ buffered formalin and embedded in paraffin. Smears and sections were stained according to a modification of the Grocott silver methenamine procedure. ${ }^{1+}$

Detection of pneumocystis by indirect immunofluorescence-Smears from lavage fluid and sputum samples that had been fixed were prepared and postfixed on microscope slides with a mixture of methanol and acetone in equal proportions for 10 minutes. To remove proteins covering antigenic sites of the parasites the smears were treated with trypsin $(2.5 \mathrm{~g} / \mathrm{l})$ in phosphate buffered saline $\mathrm{pH} 7 \cdot 1$ for $15-30$ minutes at $37^{\circ} \mathrm{C}$. Staining was performed with $3 \mathrm{~F} 6$ mouse antibody ascites specific for pneumocystis diluted 1 in 200 and sheep antimouse immunoglobulin conjugated to fluorescein isothiocyanate (fluorescence: protein ratio $(\mathrm{mol} / 1) 3 \cdot 2, \mathrm{NBL}$, Stockholm $).{ }^{13}$ The slides were examined with a Leitz orthoplan fluorescence microscope equipped with narrow band filters for fluorescein (exciting filter BP450-490, beam splitting mirror RKP510, and barrier filter LP515). All specimens were evaluated by one or both of two observers $A B$ and $\mathrm{KE}$ ) who did not know the results of the silver methenamine staining.

Serum samples-Serum samples were examined from only the 25 patients in the study group. A sample was collected as soon as pneumocystis pneumonia was clinically suspected and a second sample one to three weeks later.

Measurement of reactivity of serum to pneumocystis by indirect immunofluorescence-Paraffin sections of human lung tissue infected with $P$ carinii were used as the antigen. ${ }^{17}$ The tissue had been fixed in Bouin's trinitrophenol formol fixative. The sections $(3-5 \mu \mathrm{m})$ were mounted on to microscope slides, cleared of paraffin, rehydrated, and then rinsed for 10 minutes in phosphate buffered saline. They were incubated with $50 \mu \mathrm{l}$ of patients' serum in twofold serial dilutions for 30 minutes and washed in phosphate buffered saline three times for five minutes. Bound antibodies to pneumocytis were detected with sheep antihuman IgG conjugated to fluorescein isothiocyanate (fluorescence: protein ratio $(\mathrm{mmol}) 3 \cdot 4, \mathrm{NBL})$.
Clinical evaluation - We studied the clinical records of the 25 patients in the study group retrospectively. Cough, fever, and duration of symptoms were recorded. Dyspnoea and arterial oxygen tension could not be evaluated as they had either not been recorded or not been performed in all patients. Chest $x$ ray films were analysed, and diffuse interstitial or alveolar infiltrates, or both, present within one week before lavage were considered to be a positive finding. Chemotherapy at the time of lavage was also recorded and included curative treatment with trimethoprim and sulphamethoxazole or pentamidine or experimental prophylaxis with pyrimethamine.

\section{Results}

Table I compares the detection of pneumocystis in bronchoalveolar lavage fluid from all 65 patients by staining with silver methenamine and by indirect immunofluorescence with specific monoclonal antibody. Pneumocystis was detected in 19 samples (all from the patients positive for HIV), in 17 by both methods, and in two by labelling with the monoclonal antibody alone; in one of these last two cases only two clusters of thin walled cysts were seen, and in the other scattered free trophozoites and a couple of cysts. These results were significantly different $(\mathrm{p}<0.001$ Fisher's exact test). Pneumocystis was not detected by either method in the two groups of patients negative for HIV antibody.

TABLE I-Detection of pneumocystis in samples of bronchoalveolar lavage fluid by staining with silver methenamine and immunofluorescence with monoclonal antibody $3 F 6$. Figures are numbers of patients

\begin{tabular}{lccc}
\hline & $\begin{array}{c}\text { Immuno- } \\
\text { suppressed } \\
\text { patients } \\
\text { positive for } \\
\text { HIV antibody } \\
(\mathbf{n}=25)\end{array}$ & $\begin{array}{c}\text { Patients negative for HIV } \\
\text { antibody }\end{array}$ \\
\cline { 3 - 4 } & $19+$ & $\begin{array}{c}\text { Immuno- } \\
\text { suppressed } \\
(\mathbf{n}=18)\end{array}$ & $\begin{array}{c}\text { Immuno- } \\
\text { competent } \\
(\mathbf{n}=22)\end{array}$ \\
\hline $\begin{array}{l}\text { Pneumocystis present } \\
\text { Pneumocystis absent }\end{array}$ & 6 & 0 & 0 \\
\hline
\end{tabular}

*Presenting with undiagnosed interstitial disorders of lung.

†Pneumocystis detected in only 17 patients by staining with silver methenamine.

Staining by silver methenamine alone showed pneumocystis in six of 16 samples of bronchial brushings and nine of 14 transbronchial biopsy specimens from the 19 patients positive for HIV antibody in whom the organism was detected in lavage fluid. In the six patients positive for HIV antibody but without detectable pneumocystis in the lavage fluid silver staining of bronchial brushings from four patients and biopsy specimens from two gave negative results. Similarly, silver staining of bronchial brushings from six of the 18 immunosuppressed and 13 of the 22 immunocompetent controls and of biopsy specimens (5/18 and $3 / 22$, respectively) yielded negative results.

During six months' follow up pneumocystis pneumonia was diagnosed in only one of the six patients with negative results on examination of lavage fluid. He recovered without treatment but five and a half months later presented with severe clinical symptoms of pneumocystis pneumonia. Specific chemotherapy was started before bronchoscopy, and pneumocystis was clearly shown in the lavage fluid as well as in the sputum.

Table II summarises the clinical features, the findings on chest radiography, and the presence of antibodies to pneumocystis in the 25 patients positive for HIV antibody. In all 19 patients positive for pneumocystis, it was the first episode of pneumocystis pneumonia. The parasites were recognised by immunofluorescence despite chemotherapy having been started before bronchoscopy in 12 of the 19 


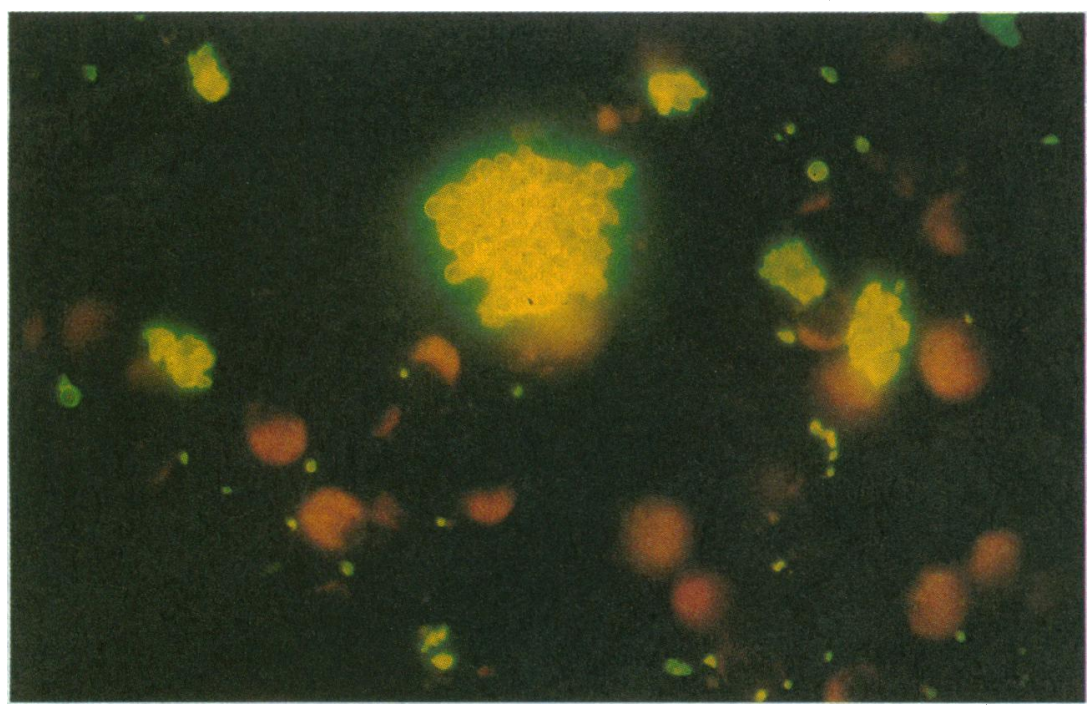

Clusters of cysts and free trophozoites of Pneumocystis carinii in bronchoalveolar lavage fluid stained by immunofluorescence with $3 F 6$ monoclonal antibody

patients. Chest $x$ ray films obtained within one week before lavage showed positive findings in 17 of the 19 patients positive for pneumocystis but in only one who was negative. Antibody to pneumocystis was recorded in only five of 18 confirmed cases of pneumocystis pneumonia: one patient had high titres (320 and 640) of the antibody, another had an initial titre of 80 but was negative for antibody two weeks later, and the remaining three had constant titres of 20 .

TABLE II-Clinical findings at time of bronchoscopy and presence of antibodies to pneumocystis in patients with confirmed or suspected AIDS

\begin{tabular}{|c|c|c|c|}
\hline & $\begin{array}{c}P \text { carinii } \\
\text { detected }^{\star} \\
(\mathbf{n}=19)\end{array}$ & $\begin{array}{c}\text { P carinii } \\
\text { not detected } \\
(\mathbf{n}=6)\end{array}$ & $\begin{array}{c}\text { Total } \\
(\mathbf{n}=25)\end{array}$ \\
\hline $\begin{array}{l}\text { No of patients with history of cough } \\
\text { No of patients with history of fever } \\
\text { Mean duration (range) of symptoms } \\
\text { (days) }\end{array}$ & $\begin{array}{c}18 \\
15 \\
22(5-50)\end{array}$ & $\begin{array}{c}6 \\
4 \\
24(9-100)\end{array}$ & $\begin{array}{c}24 \\
19 \\
23(5-100)\end{array}$ \\
\hline $\begin{array}{l}\text { No of patients receiving } \\
\text { chemotherapyt } \\
\text { No of patients with positive findings }\end{array}$ & 13 & 2 & 15 \\
\hline $\begin{array}{l}\text { on chest radiography } \ddagger \\
\text { No of patients with pneumocystis } \\
\text { antibodies } \int \text {. }\end{array}$ & $5 / 18 \|$ & 1 & 18 \\
\hline
\end{tabular}

*In bronchoalveolar lavage fluid by immunofluorescence.

†Trimethoprim sulphamethoxazole or pentamidine given to 14 patients and pyrimethamine given as prophylaxis at time of bronchoscopy for one of 13 patients positive for $P$ carinii.

positive for $P$ carinii. †Pulmonary technetium scintigram was positive in an additional patient.
〔Antibody titres $\geqslant 20$ by indirect immunofluorescence with human lung tissue

infected with pneumocystis as antigen.
||Serum from one patient not available.

Pneumocystis was identified in the sputum of patients positive for HIV antibody by immunofluorescence in 14 of 43 episodes of suspected pneumocystis pneumonia and in three of these 14 by staining with silver methenamine. The samples from the 29 remaining episodes yielded negative results by both methods. Induced sputum yielded pneumocystis in six of the 10 episodes for which specimens were available. Routine sputum samples obtained in the remaining 33 episodes showed the organism in eight cases. In 17 of the 25 patients in the study group sputum was collected within two weeks before or two days after lavage. In only four of the 17 induced sputum was obtained. Positive results were obtained in both sputum and lavage fluid in eight patients, in lavage fluid but not sputum in six, and in neither in three. By comparison with the examination of lavage fluid the sensitivity of examination of sputum was $57 \%(8 / 14)$ and the negative predictive value (true negatives/(true + false negatives)) $33 \%(3 / 9)$.

\section{Discussion}

Immunofluorescence staining with 3F6 monoclonal antibody seemed more sensitive than the conventional cytochemical stain in identifying pneumocystis in bronchoalveolar lavage fluid, thus confirming our preliminary results. ${ }^{12}$ Two samples of lavage fluid yielded positive results on immunofluorescence but negative results on staining with silver methenamine. This may be due to the ability of 3F6 monoclonal antibody to detect free trophozoites and those stages of pneumocystis cysts without a well developed cyst wall (figure) and is consistent with results of immunocytochemical studies on experimentally infected rats. ${ }^{910}$ Giemsa staining also failed to show pneumocystis in these two samples, although our experience with this stain is limited, and the method is not optimal when applied to specimens that have been fixed with ethanol. The greater ease of detecting pneumocystis by immunofluorescence is a strong point in its favour.

The chest $x$ ray findings within one week before bronchoalveolar lavage correlated well with the findings on microscopy and confirmed results of a study of chest $x$ ray findings at the time of fibreoptic bronchoscopy. ${ }^{18}$ This is in contrast, however, to the reputedly poor diagnostic value of chest $x$ ray films when evaluated on admission. ${ }^{19}$ In agreement with previous studies the clinical symptoms and the presence of circulating antibodies to pneumocystis did not seem to be of diagnostic value. ${ }^{2021}$

Immunofluorescence was superior to the silver methenamine method for detecting pneumocystis in sputum, owing to the dominance of trophozoites and immature cysts in sputum. Pneumocystis was detected more commonly in induced sputum than in routine samples on immunofluorescence. Inhalation of hypertonic saline is probably the best method of obtaining a deep sputum specimen..$^{22}$ It should be emphasised that the sensitivity of $55-56 \%$ in two previous studies was based on results from induced sputum ${ }^{45}$ whereas our sensitivity of $57 \%$ was based mainly on samples that were not induced (13/17). The sensitivity would probably increase if immunofluorescence staining were performed on induced sputum. The method of sampling sputum is safe because it includes inactivation of HIV, and it is easy to perform and sensitive. Hence examination of sputum for $P$ carinii can be used to screen all patients positive for HIV antibody presenting with pulmonary symptoms, thereby lessening the increasingly heavy load on bronchoscopy units and permitting earlier diagnosis and treatment.

Immunocytological staining of pneumocystis by immunofluorescence with 3F6 monoclonal antibody seems to have several advantages compared with conventional cytochemical staining techniques such as that using Grocott's methenamine silver stain, especially when the diagnostic procedure is carried out by units with little previous experience in identifying the parasite.

We thank Drs I Julander, S Bergdahl, $K$ Lidman, $O$ Andersson, and L-G Wiman for providing the clinical specimens, Dr $M$ Willcox for his help in revising the manuscript, and E Lindgren for examining the silver stained specimens.

1 Centers for Disease Control CDC Update. Acquired immunodeficiency syndrome United States. MMWR 1986;35:2,17-21.

2 Rorat E, Garcia RL, Skolom J. Diagnosis of Pneumocystis carinii pneumonia by cytologic examination of bronchial washings. JAMA 1985;254:1950-1.

3 Broaddus CB, Dake MD, Stulbarg MS, et al. Bronchoalveolar lavage and transbronchial biopsy for the diagnosis of pulmonary infections in the transbronchial biopsy for the diagnosis of pulmonary infections in the

4 Bigby TD, Margolskee D, Curtis GL, et al. The usefulness of induced sputum in the diagnosis of Pneumocystis carinii pneumonia in patients with the acquired immunodeficiency syndrome. Am Rev Respir Dis 1986;133:515-8.

5 Pitchenik AE, Ganjei P, Torres A, Evans DA, Rubin E, Baier H. Sputum examination for the diagnosis of Pneumocystis carinii pneumonia in the acquired immunodeficiency syndrome. Am Rev Respir Dis 1986;133:226-9.

6 Dutz W, Burke BA. Cytologic diagnosis of Pneumocystis carinii. Natl Cancer Inst Monogr 1976;43:157-61. 
7 Watts JC, Chandler FW. Pneumocystis carinii pneumonitis. The nature and significance of the methenamine silver-positive "intracystic bodies." $\mathrm{Am} \mathcal{J}$ Surg Pathol 1985:9:744-51.

8 Churukian CJ, Schenk EA. Rapid Grocott's methenamine-silver nitrate method for fungi and Pneumocystis carinii. Am J Clin Pathol 1977;68: method $427-8$.

9 Lim SK, Eveland WC, Porter RJ. Development and evaluation of a direct fluorescent antibody method for the diagnosis of Pneumocystis carini infection in experimental animals. Applied Microbiologv 1973;26:666-71.

10 Milder JE, Walzer PD, Coonrod JD, Rutledge ME. Comparison of histological and immunological techniques for detection of Pneumocystis carini in rat bronchial lavage fluid. $\mathcal{f}$ Clin Microbiol 1980;11:409-17.

11 Kovacs JA, Swan JC, Shelhamer J, Gill V, Ognibene F, Parillo JE. Prospective evaluation of a monoclonal antibody in diagnosis of Pneumocystis carinii pneumonia. Lancet 1986;ii:1-3.

12 Linder E, Elvin K, Björkman A, et al. Monoclonal antibody to detect Pneumocystis carinii. Lancet 1986;ii:634.

13 Linder E, Lundin L, Vorma H. Detection of Pneumocystis carinii in lungderived samples using monoclonal antibodies to an $82 \mathrm{kDa}$ parasite component. F Immunol Methods 1987;98:57-62.

14 Martin LS, McDougal JS, Loskoski SL. Disinfection and inactivation of the human T lymphotropic virus type III/lymphadenopathy-associated virus. human T lymphotropic vir 1985 is $152: 400-3$.

15 Spire B, Barre-Sinoussi F, Montagnier L, Cherman JC. Inactivation of lymphadenopathy associated virus by chemical disinfectants. Lancet 1984 ;ii: $899-901$.

16 Musto L, Flanigan M, Elbadawi A. Ten minute silver stain for Pneumocystis carinii and fungi in tissue sections. Arch Pathol Lab Med 1982;106:292-4.

17 Novoslawski A, Brzosko WJ. Indirect immunofluorescence for serodiagnosis of Pneumocystis carinii infection. Bulletin de l'Académie Polonaise des Sciences 1964;12:143-7.

18 Mones JM, Saldana MJ, Oldham SA. Diagnosis of Pneumocystis carinii pneumonia. Chest 1986;89:522-6.

19 Farthing CF, Shanson DC, Gazzard BG. The acquired immunodeficiency syndrome: problems associated with the management of Pneumocystis carinii pneumonia. F Infect 1985;11:103-8.

20 Walzer PD, Perl DP, Krogstad DJ, Rawson PG, Schultz MG. Pneumocystis carinii pneumonia in the United States. Ann Intern Med 1974;80:83-93.

21 Maddison SE, Walls KW, Haverkos HW, Juranek DD. Evaluation of serologic tests for Pneumocystis carinii antibody and antigenemia in patients with acquired immunodeficiency syndrome. Diagn Microbiol Infect Dis 1984;2:69-73.

22 Leilop L, Garret M, Lyons $H$. Evaluation of technique and results for obtaining sputum for lung carcinoma screening. Am Rev Resp Dis 1961;83:803-7.

(Accepted 2 March 1988)

\title{
Asian mothers' risk factors for perinatal death-the same or different? A 10 year review of Leicestershire perinatal deaths
}

\author{
Michael Clarke, David G Clayton, Elizabeth S Mason, John MacVicar
}

\begin{abstract}
A case-control study of all perinatal deaths in Leicestershire was established in 1976. By 1985 some 1342 singleton perinatal deaths had occurred. Perinatal mortality among patients of Asian origin was consistently higher than that among European women. Many of the sociomedical risk factors for perinatal death known at booking were common to both population groups. In this population of Asian women, however, low social class was not associated with perinatal risk and illegitimacy hardly ever occurred. In contrast, previous infertility among the Asian women was associated with risk of perinatal death, while no such association was found with European women. In $19 \%$ of perinatal deaths care was either inadequately provided or taken up.

The case-control design in these circumstances provides a practicable way to evaluate causal factors and at the same time to provide information of value to educators and health service planners.
\end{abstract}

\section{Introduction}

Since the 1930s the obstetric services of Britain have monitored maternal and perinatal mortality with a series of surveys and reviews. ${ }^{1.3}$ Rutstein has argued that with this method of simply reviewing the factors associated with these rare deaths-or, as he called them, "the airplane crashes in health"-we have a system for measuring quality of care that is far simpler and less costly than monitoring entire populations of pregnant women. ${ }^{+}$Nevertheless, the uncontrolled case reviews of maternal deaths have one major limitation. Although some risk factors may appear to be obvious, the degree and extent of putative risk factors in the women who do not die are often unknown or difficult to obtain and consequently the size of the mortality risks are difficult, if not impossible, to estimate. The 1958 and 1970 national perinatal mortality surveys overcame these difficulties by relating the perinatal deaths that occurred over the period of review to the total populations of births from which the deaths were derived. ${ }^{23}$

More recently the Social Services Committee of the House of Commons held an inquiry into perinatal and neonatal mortality which sought to explain why England and Wales had a higher perinatal mortality rate than many developed countries. One of the committee's recommendations was that district health authorities should pilot confidential inquiries into perinatal mortality at district level.

Since the mid-1970s we have described the causes and extent of our local perinatal mortality in much more detail than was routinely available. ${ }^{6}$ We had four objectives: (a) to measure the extent to which established risk factors, such as high parity and low social class, were risk factors in our locality; $(b)$ to measure the extent to which other factors, such as the provision of medical services and demographic changes, might contribute to the risk of perinatal mortality; (c) to estimate the extent to which avoidable factors might be detected in the case histories of the perinatal deaths; and $(d)$ to contribute our findings to the educational and planning processes of the local health services.

\section{Method}

SAMPLE POPULATION

In 1981 the population of Leicestershire was estimated to be 845000 , of whom about 60000 were of Asian origin. The Asian population (roughly $60 \%$ Hindu, 25\% Moslem, and 15\% Sikh) live predominantly within the city of Leicester. They came mainly from India (Gujerat and Punjab), Kenya, and Uganda. In 1981 women born in the New Commonwealth and Pakistan were responsible for $13 \%$ of all Leicestershire births and $32 \%$ of births in Leicester city.

The results below relate to the 1342 perinatal deaths in singleton deliveries to Leicestershire women from 1976 to 1985.

\section{STUDY DESIGN}

We used a case-control study design with cases defined as perinatal deaths occurring to women whose place of residence was Leicestershire, regardless of the place of delivery. Controls were selected as the next live birth to a Leicestershire woman in the intended place of delivery of the perinatal death to which the case related. The study was, therefore, a stratified case-control study, and estimates of relative risk and associated significance tests were calculated with the method of Mantel and Haenszel. ${ }^{7}$ Case note reviews, 